\title{
A Simpler, Cheaper and Quicker Method to Study Somatic Chromosomes from Goat, Capra hircus (L.)
}

\author{
Nandan Kumar Mondal ${ }^{*, 2)}$ and Samar Chakrabarti ${ }^{1)}$ \\ Cancer Cytogenetics Unit, Department of Zoology, The University of Burdwan, Burdwan 713 104, W.B., India
}

Received July 17, 2007; accepted October 19, 2007

\begin{abstract}
Summary A modified method for the preparation of somatic chromosomes from goat (Capra hircus) has been introduced. The method is simpler, cheaper and quicker to all the existing protocols. The material is easily available at a low cost and the method produces almost equally effective results within a short period of time. Therefore, this material and the method have been recommended both for classroom demonstration of somatic chromosomes and research. In essence, the model describes the use of HBSS as incubation medium (replacing costly culture media) for bone marrow cells collected from slaughtered goat and incubated in HBSS in presence of a metaphase arrester (colchicine) for varying length of time to standardize the optimum dose. The cells were collected by centrifugation, suspended in hypotonic solution followed by fixation in an appropriate chromatin fixative. The slides were prepared by flame dry technique, stained in Giemsa solution for observation. The diploid number has been conformed as 60 acrocentric chromosomes of varying length. Construction of karyotype revealed a heteromorphic sex chromosome status, in male $2 n=60, \mathrm{XY}$ and in female $2 n=60, \mathrm{XX}$. Spontaneous ploidal variation and the occurrence of Robertsonian fusion have been recorded. The advantage of this modified 'pocket friendly' technique has been discussed.
\end{abstract}

Key words Goat chromosome, Diploid number, Hank's Balanced Salt Solution (HBSS), Robertsonian fusion.

The study of chromosomes from somatic and germinal cells is of continuous importance both from academic and applied point of view. This is particularly important for domesticated and farm animals which constitute the Nations economic bases. Moreover the increasing cost of conventional animal models (rats and mice) for class room demonstration and for conducting different experiments has made it necessary to explore the possibility of alternative animal species. In order to introduce any animal as a model for cytogenetic study, the following conditions are needed to be fulfilled: i) easy availability and easy maintenance, ii) should be economic, iii) must be available round the year, iv) must have tissues with high proliferating capacity, v) must have a suitable karyotype, vi) should be a sensitive indicator of clastogenic, mutagenic, and carcinogenic agents and vii) must be able to convert promutagens and/or procarcinogens into its active or ultimate forms. At present, Grasshopper (Oxya velox), Swiss albino mouse (Mus musculus), albino rat (Rattus norvegicus) and Hamsters are used as model for chromosome study in the practical classroom as well as in research laboratories. Increasing use and limitless exploitation of grasshopper in the class room, excessive use of pesticides in paddy fields have made it unavailable in many localities and when it is available it costs too high ( $\$ 0.5$ only) to afford particularly to the students of developing countries. Different Rodent species used now a days to demonstrate in front of the students are not pocket friendly at all ( $\$ 1$ only per adult rat or mouse) and for a class comprising 50 students the cost would be minimum $\$ 50$ only. The paucity of fund due to budget crunch (Husnain, S.E. 2006) in most states has forced

* Corresponding author, e-mail: nandan_gm@yahoo.com, samar_c10@yahoo.co.in

Present address: 1) 44A/4/1A, C.N. Roy road, Kolkata-700039, WB. India

2) Endocrinology Laboratory, CNCI, Kolkata-700026, WB. India 


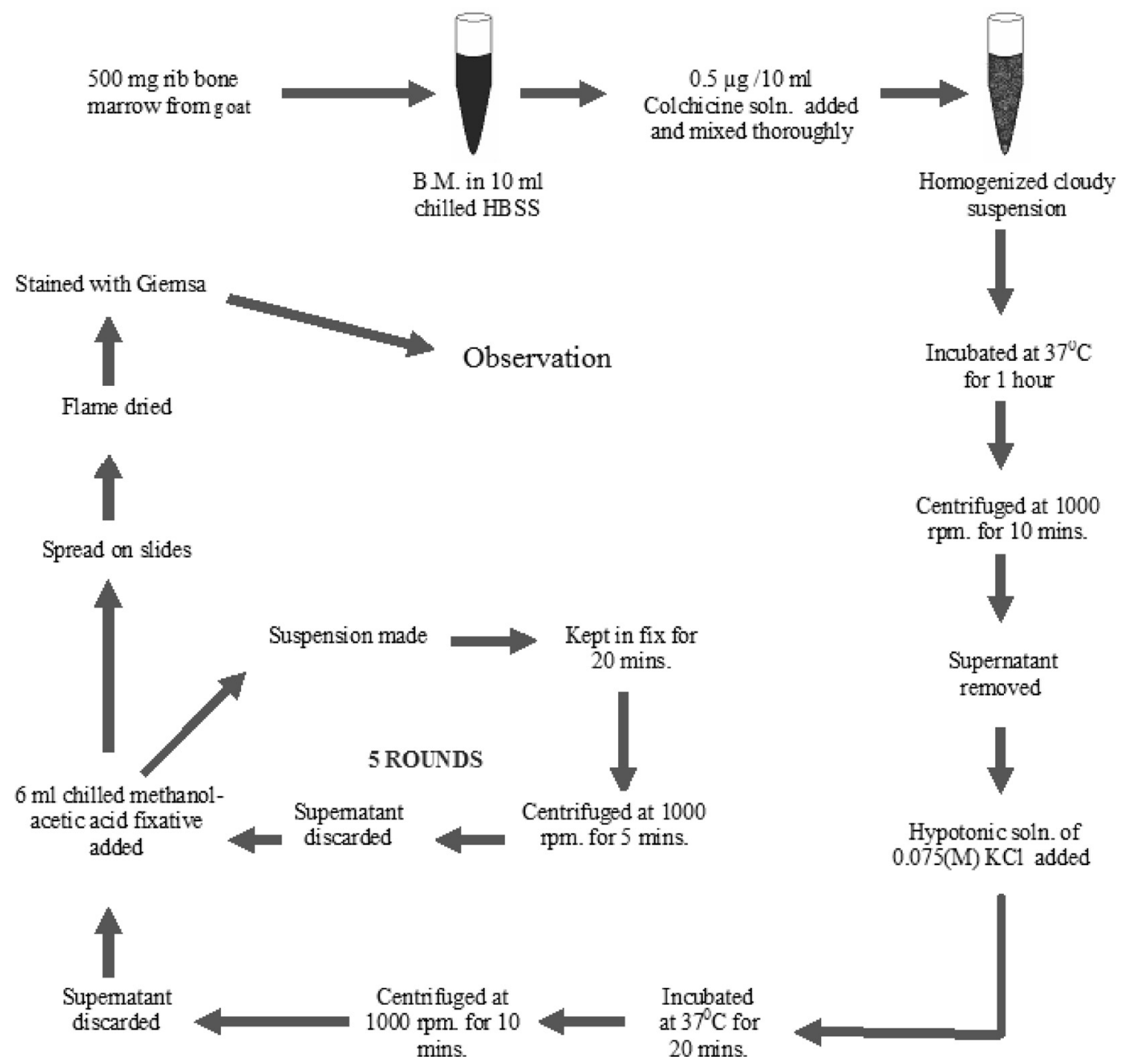

Fig. 1. Flow chart depicting the modified process of somatic chromosome preparation form goat rib bone.

the authority to reduce the grant and at the same time to increase the student intake under the political pressure or on local demand.

\section{Materials and methods}

The common Bengal variety goat, Capra hircus (Order: Artiodactyla, Family: Bovidae, Subfamily: Caprinae, Genus: Capra, Species: Capra hircus) constituted the material for the present study. In brief: small 4 inch pieces of rib bones were collected from just slaughtered healthy goat as material and kept in cold $\left(8^{\circ} \mathrm{C}\right)$ HBSS in an ice box and transported to the laboratory as quickly as possible. The marrow was extracted by pressing the tips of the rib bones with the help of a bone cutter. The bone marrow was flushed gently in HBSS along with $0.04 \%$ colchicine $(0.5 \mu \mathrm{g} / 10 \mathrm{ml}$ HBSS) as metaphase arrester and incubated for an hour in an incubator at $38^{\circ} \mathrm{C}$. The suspension was then centrifuged at $1000 \mathrm{rpm}$. for $10 \mathrm{~min}$. The supernatant was discarded and the palette was resuspended in $75 \mathrm{mM}$ potassium chloride (Analar) solution for hypotonic exposure and kept in an incubator at $37^{\circ} \mathrm{C}$ for $20 \mathrm{~min}$. The material was then centrifuged, supernatant was discarded and the palette was fixed slowly in chilled methanol-acetic acid $(3: 1 \mathrm{v} / \mathrm{v})$ fixative and kept in refrigerator 


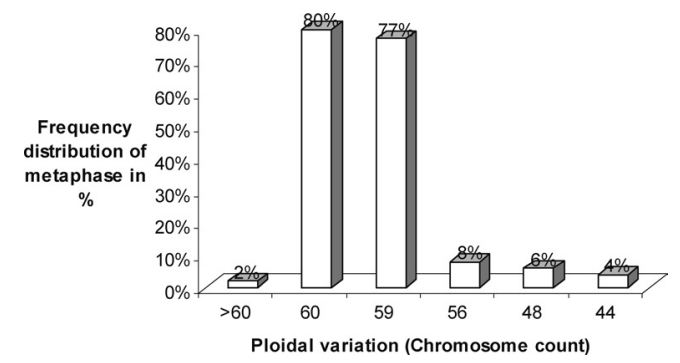

Fig. 2. Frequency distribution of dividing cells with ploidal variation in bone marrow of goat.
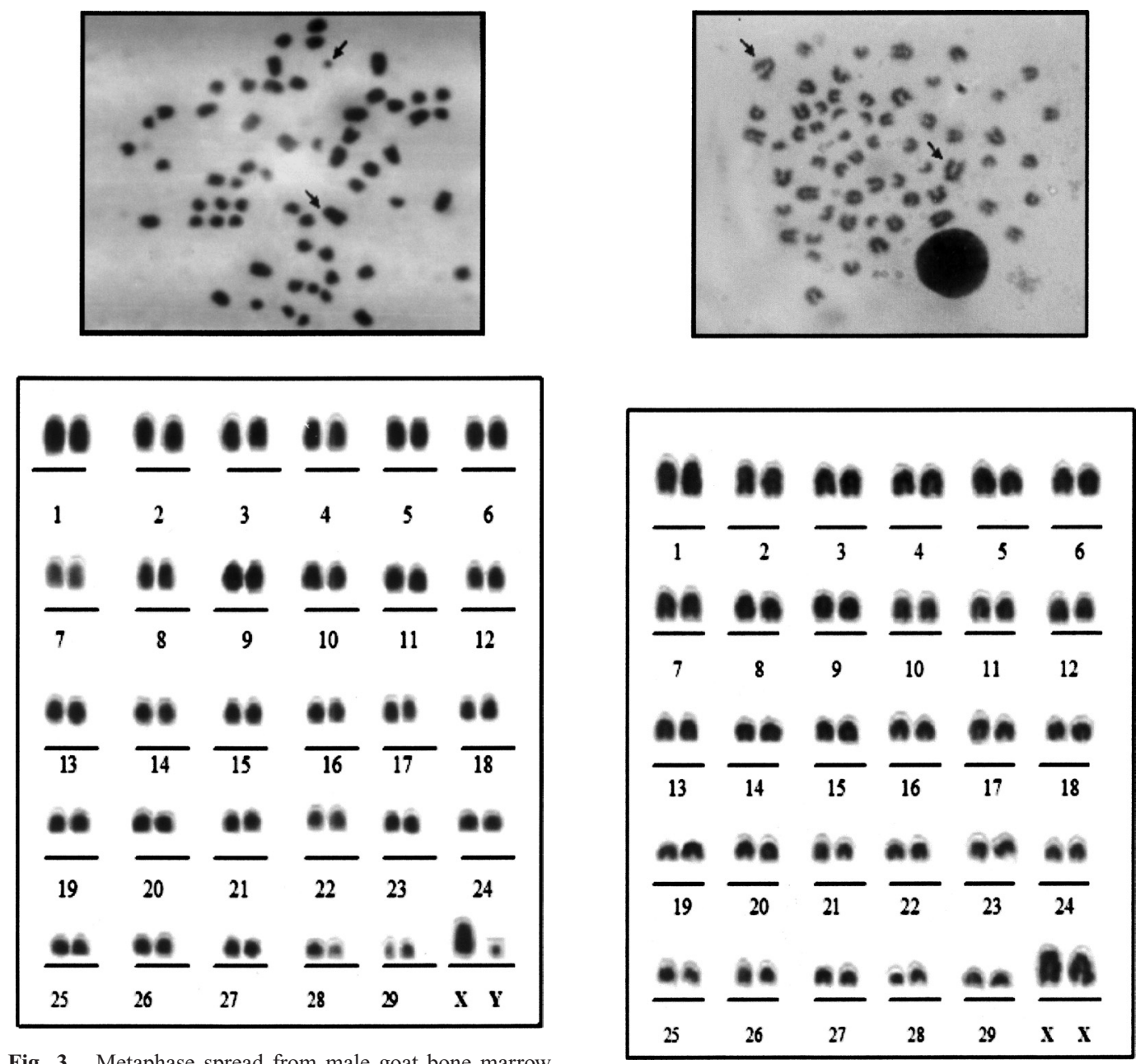

Fig. 3. Metaphase spread from male goat bone marrow cells showing $2 n=60$ acrocentric chromosomes. The arrow indicates the position of sex chromosomes. b) Karyotype constructed from above plate.

Fig. 4. Metaphase spread from female goat bone marrow cells showing $2 n=60$ acrocentric chromosomes. The arrow indicates the position of sex chromosomes. b) Karyotype constructed from above plate. 


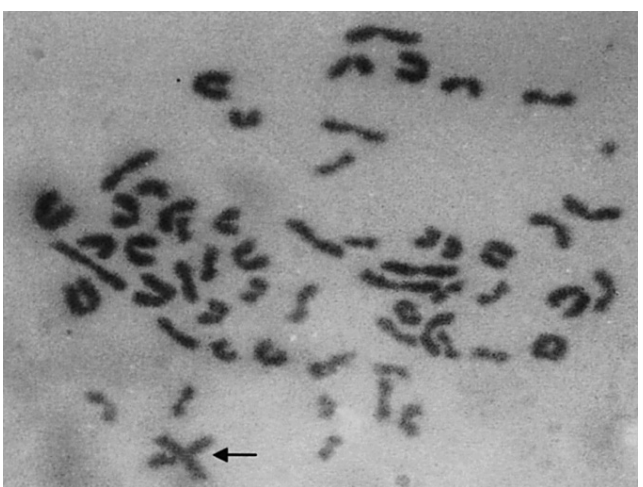

Fig. 5. Metaphase spread from male goat $(2 n=59)$ with single element with Robertsonian translocation (arrowed).

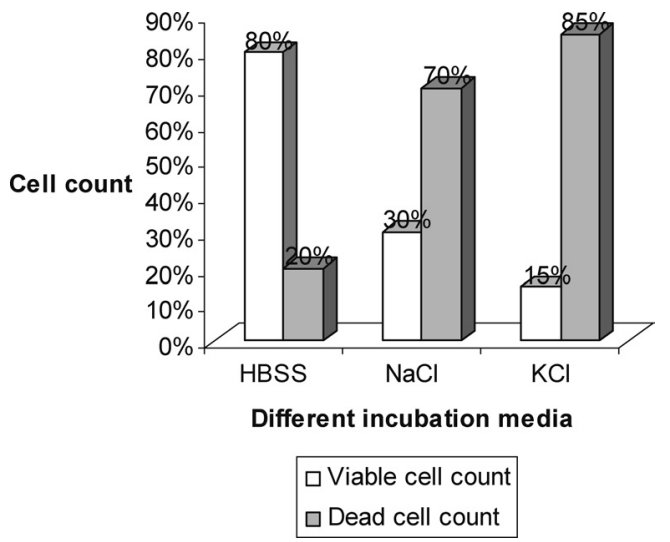

Fig. 7. Frequency distribution of dead cell count in trypan blue exclusion assay showing the advantages of using HBSS as incubation media.

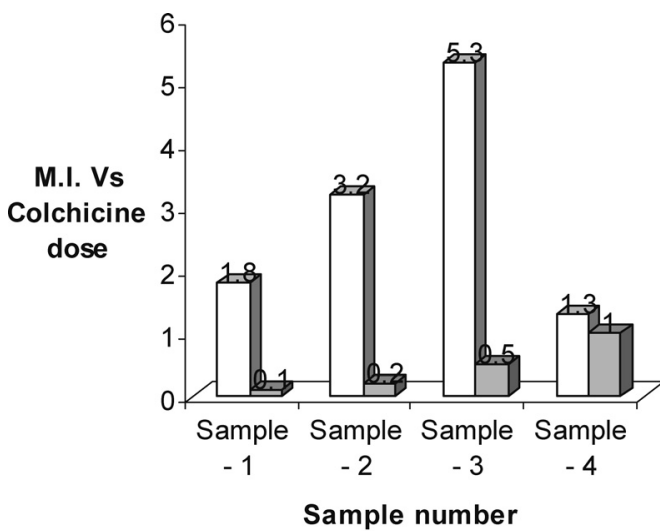

$\square$ Mitotic index
$\square$ Dose of Colchicine (micro
gm. $/ 10 \mathrm{ml}$

Fig. 6. A comparison of the effectiveness of four different doses of colchicine in arresting cells at metaphase in goat bone marrow.

for $20 \mathrm{~min}$. The process was repeated for 5 rounds. The properly fixed cells were spread on clean grease-free slides by conventional air dry technique (Chakrabarti et al. 1978, 1993, Holmquist et al. 1987). The cells spread on the slide were stained in Giemsa stain, diluted in phosphate buffer at $\mathrm{pH} 6.8$. The entire process has been depicted in the form of flow chart in Fig. 1.

\section{Observation}

Properly stained cells were then observed under microscope (Leica DM LB2 with digital camera Leica DFC 320) using 10X eyepiece and 100X oil immersion planachromatic lens. An examination of 1000 well spread metaphases from 10 male and 10 female goats revealed the most frequent chromosome count in this species was 60 represented by about $80 \%$ metaphases. A considerable number of metaphases were scored having variable number of chromosomes ranging from hypohaploidy to hyper-tetraploidy. The frequency distribution of cells with ploidal variation has been depicted in the Bar diagram (Fig. 2).

A careful observation of diploid cells revealed that all the chromosomes were of identical shape with a near terminal centromere. They differ in their respective size which varied from $7.2 \mu$ to $2.8 \mu$ depending on the degree of chromatid condensation. The photomicrographs of both male and female chromosomes have been exhibited in Figs. 3 and 4.

Male animals with 58 autosomes and a single $\mathrm{X}$ chromosome and only one Y chromosome while female with the similar number of autosomes but there were $2 \mathrm{X}$ chromosomes. On the basis of their individual length and location of centromere they were grouped into 30 different pairs. Only one pair was found to be heteromorphic and highly variable in size in case of male goat. This het- 
Table 1. A comparative account of advantages and disadvantages of the peripheral blood lymphocyte culture and HBSS incubation method of goat

\begin{tabular}{|c|c|}
\hline Peripheral blood lymphocyte culture & Goat bone marrow cell culture \\
\hline 1. Technique is time consuming $(>72 \mathrm{~h})$. & 1. Present protocol needs only $6 \mathrm{~h}$ to get result. \\
\hline $\begin{array}{l}\text { 2. Material and Technique too costly. } \\
\text { (\$100 for } 50 \text { students). }\end{array}$ & $\begin{array}{l}\text { 2. Material cost is meager ( } \$ 0.1 \text { for } 50 \text { students) } \\
\text { and technique is pocket friendly. }\end{array}$ \\
\hline $\begin{array}{l}\text { 3. Technique needs sophisticated instrumental support, } \\
\text { costly chemicals and man power. }\end{array}$ & $\begin{array}{l}\text { 3. Needs very little instrumental support and } \\
\text { man power. }\end{array}$ \\
\hline $\begin{array}{l}\text { 4. Chance of frequent culture failure due to contamination } \\
\text { or infection by fungus and bacteria. }\end{array}$ & 4. There is no/little chance of culture failure. \\
\hline
\end{tabular}

eromorphic pair seems to be the sex chromosome pair with the longest element being $\mathrm{X}$ and the smallest element was Y. But in case of female all 30 chromosome pairs were homomorphic with the longest pair being $\mathrm{X}$ chromosome i.e. one pair of $\mathrm{X}$ chromosomes. Karyotype was constructed on the basis of their relative length of arms (Levan and Sandberg 1964) with the first, the longer one and the last the smallest one. In only one male individual it was noted that chromosome count was 59 instead of usual 60 , but the fundamental number of arms remain unchanged $(2 n=59$, N.F. $=60)$, Fig. 5.

\section{Results}

There are at least 5 different essential items needed for the preparation of somatic chromosomes from any vertebrates, i) a suitable tissue as the source of dividing cells, ii) a mitogen generally PHA (phytohematogglutinin), iii) a metaphase arrestor e.g., colchicine, iv) a culture media to bathe the cells for some crucial hours before harvest and v) fixation in an appropriate fixatve. The optimum dose of metaphase arrester (colchicine) for the present cell suspension was determined by comparing the metaphase index obtained in different experimental set up and found to be $0.5 \mu \mathrm{g}$ in $10 \mathrm{ml}$ of HBSS (Fig. 6).

The other thing which needs consideration is the media used in the experiment. In an in vitro condition using peripheral lymphocytes as source of nucleated cells, the cells were cultured in appropriate culture media i.e., Eagle-99 or RPMI in presence of a mitogen, PHA/phytohemagglutinin (a costly compound of plant origin). The medium was then supplemented by colchicine or colcemid to arrest and accumulate mitotic cells at metaphase. In the present study the procedure was made simpler, cheaper and quicker by deleting 3 different costly items viz, Cell culture media, PHA and trypsin (often needed to remove anchorage dependent cells from culture vials). The total time needed to complete the entire procedure is about $5-6 \mathrm{~h}$ in the present study (from the time of collection of marrow to visualize a stained slide). The lymphocyte culture technique may need $72-78 \mathrm{~h}$ and sometimes even more. A comparison of the $2 \mathrm{viz}$, the conventional lymphocyte culture technique and the present protocol has been given in Table 1 .

\section{Discussion}

It is important to know that the location of bone marrow from where it was taken for chromosome preparation. Not all bone marrow cells were found suitable for the study. In the present finding it was noted that marrow from long bones viz., femur and humerus are not at all good source, the tiny and flat rib bones, instead was the best source which contains less fat than the marrow of the long bones. Therefore, in the present study only marrow from the rib bones was taken for processing and the entire description of the text is based on the observations made on the rib bone marrow of the goat. 
Cytogenetic studies on goat chromosome were made earlier by different workers. But most of these studies were made from peripheral blood cell culture after stimulation by a mitogen. This is for the first time we have used HBSS as incubation media for bone marrow cells in presence of mitotic poison. So far as we are aware no such study was made by any previous workers using HBSS only as incubation medium for Goat bone marrow. The use of HBSS has made it possible to allow cells to bathe for as long as $24 \mathrm{~h}$ with out any detectable deformity. The incubation of cells in $\mathrm{KCl}$ or in normal saline was unable to produce a condition provided by HBSS.

Three different incubation media used in the present experiment were: HBSS, normal saline and $\mathrm{KCl}$. Of these 3 different media in which the bone marrow cells were bathed or incubated before harvesting and fixation, the materials kept in HBSS yield the best result with relatively long survival of the cells as indicated by low dead cell count in 'Trypan blue exclusion assay' with their intact morphology. The frequency of dead cell count in this assay was also significantly less in HBSS, compared to the other two samples viz., normal saline $(0.9 \% \mathrm{NaCl})$ and $75 \mathrm{mM} \mathrm{KCl}$ solution (Fig. 7).

HBSS being a balanced salt solution not only provided an isotonic condition to the cells but also provided an energy source, nutrition, an appropriate osmotic pressure bearing environment for some longer duration to the cells.

The possible role of balanced salt solution in the present experiment is multi-faceted:

- as an irrigating, transporting and diluting tissue fluid while maintaining intra- and extra-cellular osmotic balance;

- provides cells with water and certain bulk inorganic ions essential for normal cell metabolism;

- combined with a carbohydrate, such as glucose, constitutes the principle energy source for cell metabolism; and

- cprovides buffering system to maintain the medium within the physiological $\mathrm{pH}$ range (7.2-7.6).

The present study confirms the pioneering report of Khan and Manna (1969) and Buckland and Evans (1978) who by the help of squashing seminiferous tubules and peripheral lymphocyte culture technique, respectively reported the $2 n$ number of goat was 60 . Interestingly, in the present study a good number of metaphases were recorded in one individual in whom there was a single biarmed chromosome resembling a typical Robertsonian fusion product (Padeh et al. 1971, Chakrabarti et al. 1977, 1978, Gonçalves et al. 1992). Our newly introduced simplified and cheaper methodology also corroborated the earlier studies and confirmed the result of Khan and Manna who first reported $2 n$ number by orcein staining technique, and also the result of Buckland and Evans who reported the same $2 n$ number for the species by the help of advanced (but expensive peripheral blood culture technique. Therefore the present authors recommend this new protocol as quicker, easier and cheaper methodology for chromosome preparation from goat and other farm animals both for classroom demonstration and for higher studies in fiuture.

\section{Conclusion}

Necessity is the mother of creation. The present effort is not an exception to this. The drastic price hike in recent years and a significant crunch in the budget of higher education, particularly in educational institutes in India and other third world Countries have made it mandatory to modify or replace some of the existing experimental protocols or to introduce new, novel methodology which is effective and at the same time budget friendly. Conventional cytogenetics is still a highly informative field, based on the analysis of chromosomes from dividing cells. The discipline flourished from an accidental entry of hypotonic exposure of cultured cells (Hsu, T.C. 1979), the application of mitogen viz., PHA (Moorhead et al. 1960), and the introduction of metaphase arrester and their 
immediate inclusion in the modified techniques. The technique has been proved immensely useful in both prognosis and diagnosis of animal and human diseases particularly those which are chromosome related. Contrary to some predictions, the advent of recombinant DNA technology did not minimize the importance of conventional Cytogenetics (Moorhead et al. 1960) rather; the availability of various molecular probes has propelled the field of cytogenetics into the next century with powerful adjunct techniques which can also be applied on goat chromosomes to explore many unexplored things hidden in the karyotype of this docile animal.

\section{References}

Chakrabarti, S. and Chakrabarti, A. 1977. Robertsonian fusion leading to the formation of stable dicentric chromosome in an ascites cell line of the mouse. Experientia 33: 1296-1297.

— and - 1978. Stable and transmissible dicentric chromosome with terminal centromeres in ascites cells of mouse sarcoma 180. Experientia 34: 1271-1273.

— and Biswas, T. 1993. Germ cell toxicity in tumour bearing mouse subjected to single therapeutic dose of cis-diamminedichloroplatinum (II). Current Science 65: 82-84.

Evans, H. J., Buckland, R. A. and Summer, A. T. 1973. Chromosome homology and heterochromatin in goat, sheep, and ox studied by banding techniques. Chromosoma 42: 383-402.

Gonçalves, H. C., Jorge, W. and Cury, P. R. 1992. Distribution of a Robertsonian translocation in goats. Small Ruminant Res. 8: 345-352.

Holmquist, G. P. and Motara, M. A. 1987. The Magic of Cytogenetic Technology. In: Obe, G. and Basler, A. (eds.). Cytogenetics Basic and Applied Aspects. Springer-Verlag., pp. 30-47.

Husnain, S. E. 2006. Biomedical research in India can have a global impact provided. Current. Science 91: 1435-1438.

Hsu, T. C. 1979. Human and Mammalian Cytogenetics: An Historical Perspective. Springer-Verlag, New York.

Khan, M. and Manna, G. K. 1969. Preparation of chromosome from spermatogonial cells of goat by squash technique. Cytologia 34: 60-64.

Levan, A., Fredga, K. and Sandberg, A. 1964. Nomenclature for centromeric position on chromosomes. Hereditas 52: 201-220.

Mark, H. F. L. and Wolman, S. R. 2000. Medical Cytogenetics. In: Mark, H. F. L. (eds.). Culture and harvest of tissues for chromosome analysis. Marcel Dekker, Inc., New York., pp. 91-120.

Moorhead, P. S., Nowell, P. C., Nellman, W. J., Battips, D. M. and Hungerford, D. A. 1960. Chromosome preparations of leukocytes cultured from human peripheral blood. Exp. Cell Res. 20: 613-616.

Padeh, B., Wysoki, M. and Soller, M. 1971. Further studies on a Robertsonian translocation in the Saanen goat. Cytogenetics 10: 61-69. 